\title{
Propensity score matching analysis of a phase II study on simultaneous modulated accelerated radiation therapy using helical tomotherapy for nasopharyngeal carcinomas
}

Lei $\mathrm{Du}^{1,2+}$, Xin-Xin Zhang ${ }^{3+}$, Lin-Chun Feng ${ }^{1}$, Bao-Lin Qu' ${ }^{1}$ Jing Chen ${ }^{1}$, Jun Yang ${ }^{4}$, Hai-Xia Liu ${ }^{5}$, Shou-Ping Xu', Chuan-Bin Xie ${ }^{1}$ and Lin $\mathrm{Ma}^{1 *}$

\begin{abstract}
Background: Using propensity score matching method (PSM) to evaluate the feasibility and clinical outcomes of simultaneous modulated accelerated radiation therapy (SMART) using helical tomotherapy (HT) in patients with nasopharyngeal carcinoma (NPC).

Methods: Between August 2007 and January 2016, 381 newly diagnosed NPC patients using HT were enrolled in pre-PSM cohort, including 161 cases in a prospective phase II study (P67.5 study, with a prescription dose of 67.5Gy in 30 fractions to the primary tumour and positive lymph nodes) and 220 cases in a retrospective study (P70 study, with a prescription dose of 70Gy in 33 fractions to the primary tumour and positive lymph nodes). Acute and late toxicities were assessed according to the established RTOG/EORTC criteria and Common Terminology Criteria for Adverse Events (CTCAE) V 3.0. Survival rate were assessed with Kaplan-Meier method, log-rank test and Cox regression.

Results: After matching, 148 sub-pairs of 296 patients were generated in post-PSM cohort. The incidence of grade 3-4 leukopenia, thrombocytopenia and anemia in the P67.5 group was significantly higher than in the P70 study, but no significant different was found in other acute toxicities or late toxicities between the two groups. The median followup was 33 months in the P67.5 and P70 group, ranging 12-54 months and 6-58 months, respectively. No significant differences in 3-year local-regional recurrence free survival (LRRFS), distant metastasis-free survival (DMFS), disease free survival (DFS) and overall survival (OS) were observed between the 2 groups. Univariate analysis showed that age, $T$ stage, clinical stage were the main factors effecting survival. Cox proportional hazards model showed that 67.5Gy/30F pattern seemed superior in 3-year OS (HR $=0.476,95 \%$ Cl: 0.236-0.957).
\end{abstract}

Conclusions: Through increasing fraction dose and shortening treatment time, the P67.5 study achieved excellent short-term outcomes and potential clinical benefits, with acceptable acute and late toxicities.

Trial registration: The trial was registered at Chinese Clinical Trial Registry on 5 July 2014 with a registration code of ChiCTRONC-14,004,895.

Keywords: Nasopharyngeal carcinoma, Intensity-modulated radiation therapy, Dose fractionation, Propensity score matching, Survival

\footnotetext{
* Correspondence: malinpharm@sina.com

${ }^{\dagger}$ Equal contributors

'Department of Radiation Oncology, Chinese PLA General Hospital, 28

Fuxing Road, Beijing 100853, China

Full list of author information is available at the end of the article
} 


\section{Background}

Currently, simultaneous modulated accelerated radiation therapy (SMART) is the most widely used intensity modulated radiation therapy (IMRT) pattern in the treatment of nasopharyngeal carcinomas (NPC) [1]. SMART can simultaneously delivery different doses to different targets and improve local control rate (LCR) through increasing fraction dose in the primary tumour and metastatic nodes and shortening the overall treatment time (OTT) to reduce post-process accelerated repopulation of tumour cells. Some studies have confirmed that SMART using Helical TomoTherapy (HT) system has significant dosimetric advantages in the treatment of NPC $[2,3]$. More than 600 NPC patients have been treated with HT system at our centre. Based on previous $70 \mathrm{~Gy} / 33 \mathrm{~F}$ pattern, we conducted in September 2011 a prospective phase II study, P67.5 study, with a prescription dose of $67.5 \mathrm{~Gy}$ in 30 fractions to the primary tumour and positive lymph nodes [4]. Due to increased fraction dose and shortened OTT, the corrected biological effective dose (BED) to the primary tumour and positive lymph nodes increased from $62 \mathrm{~Gy}$ to $62.9 \mathrm{~Gy}$, while that to late reaction tissues (LRTs) decreased from 99.7Gy to 97.9Gy $(\alpha / \beta=5 \mathrm{~Gy})$, which could theoretically improve local control rate while reducing radiation injury. The study was approved by the research ethics board of the Chinese PLA General Hospital with an official number of S2014-048-01, and with a registration code of ChiCTRONC-14,004,895. To confirm the safety and feasibility of the P67.5 study, we retrospectively analyzed the data of our previous P70 study with a prescription dose of $70 \mathrm{~Gy}$ in 33 fractions to the primary tumour and positive lymph nodes and used propensity score matching method (PSM) [5] to screen the cases and exclude the impact of confounding factors.

\section{Methods}

\section{Patient's characteristics}

From August 2007 to January 2014, 381 newly diagnosed non-metastatic NPC patients treated by HT were registered in our centre, and among them 161 cases in P67.5 study and 220 cases in P70 study. Patients' characteristics should be met the following conditions: Pathological confirmed squamous cell carcinoma; World Health Organization (WHO) types I and II; Karnofsky performance status (KPS) $\geq 70$. All patients experienced nasopharyngeal and skull base magnetic resonance imaging (MRI), endoscopic evaluation, chest CT, neck and abdomen ultrasound, and bone scanning. Positron emission tomography (PET) was optional. Clinical stage was practiced according to the Union Internationale Contre le Cancer (UICC) 2002 staging system.

\section{Propensity score matching (PSM)}

Excluding the patients affected by non-disease factors, we ultimately selected 374 cases, of whom 158 cases in
P67.5 study and 216 cases in P70 study. The PSM method was used to control the balance between the two groups and there were five covariates in the score scale including gender, age, $\mathrm{T}$ stage, $\mathrm{N}$ stage and clinical stage. According to the 1: 1 ratio, logistic regression and the nearest matching pattern were also used and 148 sub-pairs of 296 patients were generated.

\section{Helical tomotherapy (HT)}

Plain and enhanced CT images scan for treatment planning were the same in both groups using Brilliance TM CT Big Bore and the images were transmitted to the Pinnacle3 8.0 workstation and fused. According to ICRU 50 and 62 reports, Gross target volume of primary tumor (GTVnx) and metastatic lymph nodes (GTVnd) were respectively defined as the visible tumor and involved nodes. The pGTVnx was obtained by expanding the corresponding GTVnx with a margin of $3-5 \mathrm{~mm}$ while limited by the brainstem, spinal cord, optic chiasma and optic nerve. The pGTVnd was the GTVnd with an expansion of $3 \mathrm{~mm}$. Clinical target volume 1 (CTV1) covered nasopharynx, high-risk local structures (i.e., skull base, clivus, parapharyngeal space, retropharyngeal lymph nodes, sphenoid sinus, sphenomaxillary fossa, posterior part of the nasal cavity and maxillary sinus, and oropharynx), as well as positive lymph nodes and nodes at level IB (when nodes at level IIA were involved), level II and superior part of VA. Clinical target volume 2 (CTV2) included lymph nodes at level Ш, IV, VB and inferior part of VA as a prophylactic irradiated volume. Planning target volume1 (PTV1) and 2 (PTV2) were generated with a $3 \mathrm{~mm}$ margin of CTV1 and CTV2 at least $2 \mathrm{~mm}$ from skin. Enhanced MRI or PET images were used as a guide for target contours. In P67.5 study, prescription dose was delivered to pGTVnx and pGTVnd at 67.5Gy (2.25Gy per fraction), PTV1 at 60Gy (2Gy per fraction) and PTV2 at 54Gy (1.8Gy per fraction) in 30 fractions. In P70 study, prescription dose was delivered to pGTVnx and pGTVnd at 70Gy (2.12Gy per fraction), PTV1 at 60Gy (1.82Gy per fraction) and PTV2 at 54-56Gy (1.63-1.70Gy per fraction) in 33 fractions. Details of plan designing and dose-volume constraints for organs at risk (OARs) referred to our previous articles $[4,6]$. In both groups, HT plans were made by the same group of physicists with the same plan parameters using TomoTherapy ${ }^{\oplus}$ Planning Station.

\section{Chemotherapy and anti-EGFR monoclonal antibody (Mab) treatment}

Based on existing clinical evidence, radiation therapy with concurrent platinum-based chemotherapy were used as standard treatment for locally advanced NPC patients. A total of 201 patients (67.9\%) underwent concurrent chemoradiotherapy (CCRT), of whom 128 (86.5\%) in P67.5 study and 73 (49.3\%) in P70 study. Concurrent chemotherapy 
included two patterns: 1) cisplatin $80 \mathrm{mg} / \mathrm{m}^{2}$, d1, every 3 weeks; 2) cisplatin $60 \mathrm{mg} / \mathrm{m}^{2}$ and docetaxel $60 \mathrm{mg} / \mathrm{m}^{2}$, d1, every 3 weeks. Chemotherapy doses and cycles were slightly adjusted according to the adverse reactions. Many studies especially in high incidence areas have proved the value of anti-EGFR Mab treatment in NPC patients [7-9]. As early as 2010, the Chinese Version of Clinical Practice Guidelines in NPC added concurrent anti-EGFR Mab treatment as an option for T1 N1-3 and T2-T4 with any N patients. In our study, 117 cases underwent anti-EGFR Mab treatment, of whom 54 (36.5\%) in P67.5 study and 63 (42.6\%) in P70 study (cetuximab with a loading dose of $400 \mathrm{mg} / \mathrm{m}^{2}$ and then $250 \mathrm{mg} / \mathrm{m}^{2}$ or nimotuzumab $200 \mathrm{mg}$, $\mathrm{d} 1$, every week). In addition to CCRT, induction chemotherapy (ICT) and adjuvant chemotherapy (ACT) were both recommended for locally advanced NPC patients. Based on characteristics of patients, disease staging, and tolerance for the treatment with the principle of no more than 6 cycles of total chemotherapy, ICT and/or ACT were individualized used for the patients. The specific use of chemotherapy and anti-EGFR Mab treatment were shown in Table 1.

\section{Statistical analyses and follow-up}

Acute and late toxicities were assessed according to the established Radiation Therapy Oncology Group and the European Organization for Research and Treatment of Cancer (RTOG/EORTC) criteria and part of late toxicities referred to Common Terminology Criteria for Adverse Events (CTCAE) v3.0 at the same time. The follow-up started at the first day of radiation therapy and ended on January 2016, with a median follow-up of 33 months (6-58 months) and a follow-up rate of $100 \%$. Standardized differences were estimated for all baseline covariates before and after matching. In the matched data, dose comparisons were performed using $\mathrm{T}$ test and

Table 1 Chemotherapy and anti-EGFR monoclonal antibody (Mab) treatment

\begin{tabular}{|c|c|c|c|c|c|}
\hline \multirow[t]{3}{*}{ Chemotherapy } & \multirow{2}{*}{\multicolumn{2}{|c|}{$\begin{array}{l}\text { P67.5 } \\
\text { anti-EGFR Mab } \\
\text { treatment }\end{array}$}} & \multirow{2}{*}{\multicolumn{2}{|c|}{$\begin{array}{l}\text { P70 } \\
\text { anti-EGFR Mab } \\
\text { treatment }\end{array}$}} & \multirow[t]{3}{*}{ Tota } \\
\hline & & & & & \\
\hline & + & - & + & - & \\
\hline None & 3 & 11 & 20 & 19 & 53 \\
\hline ICT & 2 & 2 & 0 & 4 & 8 \\
\hline CCRT & 1 & 6 & 20 & 23 & 50 \\
\hline$A C T$ & 0 & 0 & 18 & 11 & 29 \\
\hline$I C T+C C R T$ & 29 & 32 & 0 & 8 & 69 \\
\hline CCRT + ACT & 4 & 7 & 2 & 20 & 33 \\
\hline$I C T+C C R T+A C T$ & 13 & 36 & 0 & 0 & 49 \\
\hline $\mathrm{ICT}+\mathrm{ACT}$ & 2 & 0 & 3 & 0 & 5 \\
\hline Total & 54 & 94 & 63 & 85 & 296 \\
\hline
\end{tabular}

Abbreviation: ICT induction chemotherapy, CCRT concurrent chemoradiotherapy, ACT adjuvant chemotherapy toxicities in both groups were compared with Pearson X2 test. Survival rates were assessed using the KaplanMeier method. The Log-rank test and the Cox proportional hazards model were used to identify prognostic factors independently associated with survival and to estimate hazard ratios (HR). Two-sided $p$ values of $<0.05$ were considered statistically significant. Statistical analyses were performed using SPSS software package version 22.0 (Chicago, IL, USA).

\section{Results}

\section{Patient characteristics}

Baseline patient characteristics in the pre- and post-PSM cohort were displayed in Table 2. A total of 296 eligible patients were enrolled, including 215 males and 81 females. The ratio of male to female was about 2.65:1. Mean age was 45 years, and patients in P67.5 group were slightly older than those in P70 group (45.7 vs. 44.3 years). Although no significant difference was detected for T stage in pre-PSM cohort $(p=0.485)$, significant differences were noted for $\mathrm{N}$ stage $(p=0.014)$ and clinical stage $(p=0.017)$ between the two groups. These differences were well-balanced through PSM method $(p=0.985, p=0.829$, respectively). The specific TNM stage was shown in Table 3.

\section{Dosimetric analysis}

The specific dose distributions (Table 4) showed a significant dose reduction in the maximum dose of brainstem, spinal cord, eyeballs, lens, optic nerves and the mean dose of pGTVnx, pGTVnd, PTV2, temporomandibular joint, oral cavity and larynx-esophagus-trachea in P67.5 group compared with that in P70 group. In addition, the mean dose of left and right parotid gland decreased by $0.7 \mathrm{~Gy}$ and 0.4 Gy, respectively, but without statistical significance. In our opinion, the above results were mainly because of a 2.5Gy reduction of prescription dose. However, the mean dose of PTV1 and inner ear were almost the same in both groups, which was probably because the prescription dose of PTV1 remained the same and inner ears were always involved in PTV1.

\section{Acute and late toxicities}

Acute side effects were investigated weekly and peak toxicities were recorded. Skin reactions, oral mucositis, xerostomia, pharyngo-esophagitis were still common clinical acute adverse reactions, which appeared around the $10^{\text {th }}$ fraction. The most severe oral mucositis and pharyngo-esophagitis occurred during the $20^{\text {th }}$ to $25^{\text {th }}$ fraction and then gradually relieved, but the most severe xerostomia and skin reaction generally occurred at the end of radiation therapy. Statistical analysis showed that radiation related acute toxicities were mainly grade 1 or 2 and the fractionation pattern did not significantly affect the incidence and constituent ratios. 
Table 2 Baseline patient characteristics in the pre- and post-PSM cohort

\begin{tabular}{|c|c|c|c|c|c|c|}
\hline \multirow[b]{2}{*}{ Characteristics } & \multicolumn{3}{|l|}{ Before PSM } & \multicolumn{3}{|l|}{ After PSM } \\
\hline & $\begin{array}{l}\text { P67.5 } \\
(n=158)\end{array}$ & $\begin{array}{l}\text { P70 } \\
(n=216)\end{array}$ & $P^{*}$ & $\begin{array}{l}\text { P67.5 } \\
(n=148)\end{array}$ & $\begin{array}{l}\text { P70 } \\
(n=148)\end{array}$ & $P^{*}$ \\
\hline Gender & & & 0.537 & & & 0.896 \\
\hline Male & 114 (72.2\%) & 162 (75.0\%) & & 108 (73.0\%) & 107 (72.3\%) & \\
\hline Female & $44(27.8 \%)$ & $54(25.0 \%)$ & & 40 (27.0\%) & $41(27.7 \%)$ & \\
\hline Age (years) & & & 0.434 & & & 0.444 \\
\hline Median (range) & $47(15-75)$ & $44(10-81)$ & & $47(15-75)$ & $45(10-81)$ & \\
\hline Mean (SD) & $45.5(13.5)$ & 44.4 (13.9) & & $45.7(13.0)$ & $44.3(13.8)$ & \\
\hline T stage & & & 0.485 & & & 0.822 \\
\hline 1 & 41 (25.9\%) & $63(29.2 \%)$ & & 41 (27.7\%) & 46 (31.1\%) & \\
\hline 2 & 49 (31.0\%) & 71 (32.9\%) & & $48(32.4 \%)$ & $41(27.7 \%)$ & \\
\hline 3 & $43(27.2 \%)$ & $44(20.4 \%)$ & & $34(23.0 \%)$ & $34(23.0 \%)$ & \\
\hline 4 & $25(15.8 \%)$ & $38(17.6 \%)$ & & 25 (16.9\%) & $27(18.2 \%)$ & \\
\hline N stage & & & 0.014 & & & 0.985 \\
\hline 0 & $21(13.3 \%)$ & $46(21.3 \%)$ & & $21(14.2 \%)$ & $22(14.9 \%)$ & \\
\hline 1 & $50(31.6 \%)$ & 85 (39.4\%) & & $50(33.8 \%)$ & $51(34.5 \%)$ & \\
\hline 2 & 77 (48.7\%) & 71 (32.9\%) & & 68 (45.9\%) & $65(43.9 \%)$ & \\
\hline 3 & $10(6.3 \%)$ & $14(6.5 \%)$ & & $9(6.1 \%)$ & $10(6.8 \%)$ & \\
\hline Clinical stage & & & 0.017 & & & 0.829 \\
\hline I & 7 (4.4\%) & $16(7.4 \%)$ & & $7(4.7 \%)$ & $8(5.4 \%)$ & \\
\hline$\|$ & $36(22.8 \%)$ & 72 (33.3\%) & & $36(24.3 \%)$ & 39 (26.4\%) & \\
\hline III & $80(50.6 \%)$ & $76(35.2 \%)$ & & 71 (48.0\%) & $63(42.6 \%)$ & \\
\hline $\mathrm{IVa}$ & 35 (22.2\%) & $52(24.1 \%)$ & & $34(23.0 \%)$ & 38 (25.7\%) & \\
\hline
\end{tabular}

Abbreviation: PSM Propensity score matching

* $P$-values were calculated using the Pearson $\mathrm{X} 2$ test

Hematologic toxicity was another important factor that influenced treatment compliance due to the intervention of chemotherapy. The incidence of grade 3-4 leukopenia, thrombocytopenia and anemia significantly increased in P67.5 group compared with P70 group (78.4\% vs. 10.1\%). Radiation therapy was interrupted in 11 patients (7 in P67.5 group, 4 in P70 group) due to acute toxicities with an average interruption time of 9.2 days (6-14 days). All patients finished radiation therapy except one in P67.5 group, who finally received $60.75 \mathrm{~Gy} / 27 \mathrm{~F}$ due to gastrointestinal adverse reaction. At the end of radiation therapy, patients' weight

Table 3 Distributions of patients in P67.5 and P70 study according to the UICC 2002 staging system

\begin{tabular}{|c|c|c|c|c|c|c|c|c|c|c|}
\hline \multirow[t]{2}{*}{ Stage } & \multicolumn{5}{|c|}{ P67.5 } & \multicolumn{5}{|l|}{ P70 } \\
\hline & $\mathrm{N}_{0}$ & $N_{1}$ & $\mathrm{~N}_{2}$ & $\mathrm{~N}_{3}$ & Total & $\mathrm{N}_{0}$ & $N_{1}$ & $\mathrm{~N}_{2}$ & $\mathrm{~N}_{3}$ & Total \\
\hline $\mathrm{T}_{1}$ & 7 & 17 & 15 & 2 & 41 & 8 & 19 & 15 & 4 & 46 \\
\hline $\mathrm{T}_{2}$ & 5 & 14 & 25 & 4 & 48 & 6 & 14 & 18 & 3 & 41 \\
\hline$T_{3}$ & 5 & 15 & 12 & 2 & 34 & 8 & 7 & 18 & 1 & 34 \\
\hline $\mathrm{T}_{4}$ & 4 & 4 & 16 & 1 & 25 & 0 & 11 & 14 & 2 & 27 \\
\hline Total & 21 & 50 & 68 & 9 & 148 & 22 & 51 & 65 & 10 & 148 \\
\hline
\end{tabular}

lost by $11.2 \%$ on the average without significant difference between the two groups (Table 5).

Late toxicities generally appeared three months after radiation therapy and included subcutaneous tissue fibrosis, xerostomia, otitis media, taste changes, dehisce difficulty, hearing loss, tooth and periodontal diseases (including tooth sensitivity, crown fracture, gingival recession), hypothyroidism, etc. Most of the late toxicities were grade 1 with a small number grade 2 or more toxicities. Although most of the late toxicities could be alleviated as time passed, they were still the main factors affecting the quality of life. And there was no significant difference between the two groups in the composition ratio of late toxicities (Table 5).

\section{Short-term outcomes and survival analysis}

Short-term outcomes were evaluated with Response Evaluation Criteria in Solid Tumors (RECIST, Version 1.1) within 1 to 3 months after radiation therapy. One hundred and sixteen cases (55 in P67.5 group and 61 in P70 group) developed a complete remission (CR), 156 cases (80 in P67.5 group and 76 in P70 group) had a partial remission (PR) and 24 cases (13 in P67.5 group and 11 in P70 group) 
Table 4 Mean dose of organs at risk

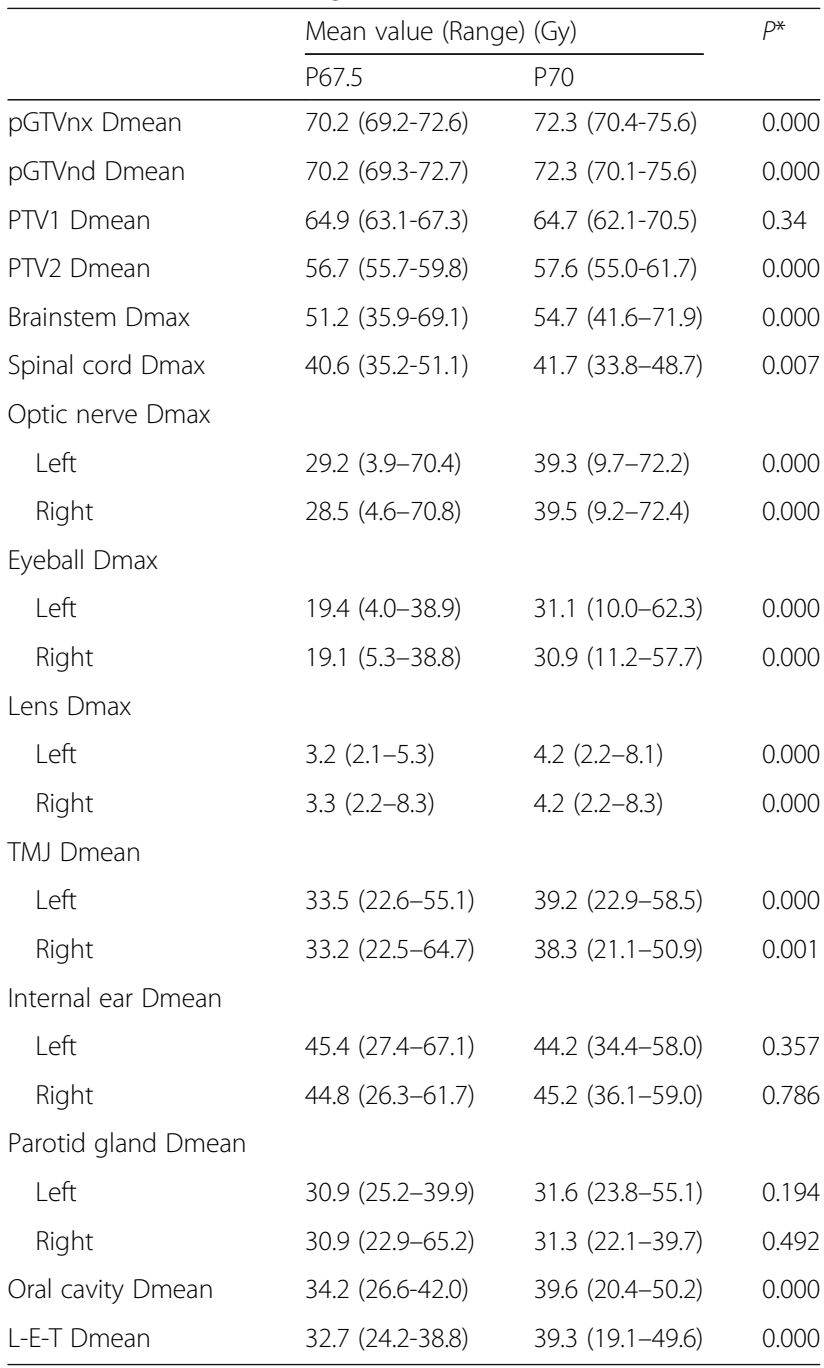

Abbreviation: Dmean mean dose, Dmax maximum dose, TMJ

Temporomandibular joint, L-E-T Larynx-esophagus-trachea

${ }^{*} P$-values were calculated using the $T$ test

had a stable disease (SD) in the primary tumour, without significant difference between the two groups $\left(\chi^{2}=0.580\right.$, $p=0.748)$. In 253 patients with metastatic nodes, 114 cases (53 in P67.5 group and 61 in P70 group) had a CR, 123 cases (63 in P67.5 group and 60 in P70 group) had a PR and 16 cases (10 in P67.5 group and 6 in P70 group) had a SD, without significant difference between the two groups either $\left(x^{2}=1.631, p=0.442\right)$. The whole effective rate was $100 \%$.

Thirty-nine patients developed treatment failure during the follow-up, including 11 local recurrences, 6 regional recurrences, 21 distant metastases, 6 hemorrhages and 1 systemic failure (Table 6). The number of local recurrent cases was similar in P67.5 and P70 group (5 cases vs. 6 cases) and the recurrence areas were mainly within the target field. The patients with local recurrent in P67.5 group had lower mortality and longer relapse- to-death time, probably due to a higher proportion of patients receiving salvage therapy (60\% in P67.5 group vs. 33\% in P70 group). Three patients had regional recurrence in each group, $2 / 3$ in P70 group were dead, while 3/3 in P67.5 group were still alive. Distant metastasis was the most common failure pattern in both groups and the most common metastatic sites were liver, bone, and lung. Whether to receive salvage treatment would determine the level of mortality for the patients of distant metastasis. Hemorrhage, a specific failure pattern, could result in a high mortality, and significantly developed more in P70 group than in P67.5 group (5 cases vs. 1 case). One patient in P70 group died of multiple-organ failure due to malnutrition.

The median follow-up was 33 months in the P67.5 and P70 group, ranging 12-54 months and 6-58 months, respectively. The 3-year local-regional relapse free survival (LRRFS) was $94.0 \%$ and $92.7 \%$, distant metastasis free survival (DMFS) was $93.2 \%$ and $91.1 \%$, disease free survival (DFS) was $88.5 \%$ and $87.8 \% \%$, and overall survival (OS) was $93.9 \%$ and $90.4 \%$, respectively, without significant difference between the two groups (Fig. 1).

Univariate analysis showed that $\mathrm{T}$ stage was an independent factor of the 3-year LRRFS ( $p=0.034)$; age was the factor affecting the 3-year DMFS $(p=0.049)$ and OS $(p=0.008)$; factors affecting the 3 -year DFS included age $(p=0.002)$, T stage $(p=0.045)$ and clinical stage $(p=0.019)$ (Table 7). Multivariate analysis was performed with Cox proportional hazard model. Age $(<50$ years vs. $\geq 50$ years) and clinical stage (I-II vs. III-IV) were the main factors affecting the 3-year DMFS $(\mathrm{HR}=2.617$ and $\mathrm{HR}=9.786)$, DFS $(\mathrm{HR}=3.058$ and $\mathrm{HR}=4.487)$ and $\mathrm{OS}(\mathrm{HR}=2.914$ and $\mathrm{HR}=4.208)$. In addition, compared with P70 group, P67.5 group had a superior 3-year OS $(\mathrm{HR}=0.476)$, and no factor affecting the 3-year LRRFS was detected (Table 8).

\section{Discussion}

HT is a kind of advanced technology of radiation therapy and the treatment model of "rotation - step in - shoot" is on behalf of a type of highly efficient and high accurate IMRT [10]. Since our centre installed the first HT unit in china in September 2007, over 3000 cases had been treated by Match 2016. The P67.5 study was a nonrandomized single-centre prospective study which aimed to evaluate the safety and feasibility of a new fractionation pattern, and the control group (P70 study) was a retrospective study with classical fractionation. In order to minimize the impact of confounding factors, we used PSM method and effectively corrected the hybrid bias in $\mathrm{N}$ stage and clinical stage. The final general characteristics of patients in both groups tended to be balanced.

The RTOG 0225 study [11] laid the fractionation of 70Gy/33F with SMART technology to become the standard IMRT pattern of NPC and the LCR reached $92.6 \%$ at 2- 
Table 5 Acute and late toxicities in the propensity-matched cohorts [n (\%)]

\begin{tabular}{|c|c|c|c|c|c|c|c|}
\hline \multirow[t]{2}{*}{ Toxicities } & \multicolumn{3}{|l|}{ P67.5 } & \multicolumn{3}{|l|}{ P70 } & \multirow[t]{2}{*}{$P^{*}$} \\
\hline & Grade 0 & Grade 1-2 & Grade 3-4 & Grade 0 & Grade 1-2 & Grade 3-4 & \\
\hline \multicolumn{8}{|l|}{ Acute toxicities } \\
\hline Skin reaction & $5(3.4 \%)$ & 137 (92.5\%) & $6(4.1 \%)$ & $5(3.4 \%)$ & 136 (91.9\%) & $7(4.7 \%)$ & 0.961 \\
\hline Mucositis & $2(1.4 \%)$ & $133(89.8 \%)$ & $13(8.8 \%)$ & $1(0.7 \%)$ & $141(95.2 \%)$ & $6(4.1 \%)$ & 0.207 \\
\hline Xerostomia & $2(1.4 \%)$ & $146(98.6 \%)$ & 0 & $7(4.7 \%)$ & $141(95.3 \%)$ & 0 & 0.091 \\
\hline Pharyngo-esophagitis & 0 & $144(97.3 \%)$ & $4(2.7 \%)$ & $4(2.7 \%)$ & $143(96.6 \%)$ & $1(0.7 \%)$ & 0.055 \\
\hline Leucopenia & $31(20.9 \%)$ & 79 (53.4\%) & 38 (25.7\%) & $58(39.2 \%)$ & $80(54.0 \%)$ & $10(6.8 \%)$ & 0.000 \\
\hline Anemia & 73 (49.3\%) & $71(48.0 \%)$ & $4(2.7 \%)$ & $137(92.6 \%)$ & $11(7.4 \%)$ & 0 & 0.000 \\
\hline Thrombocytopenia & 118 (79.7\%) & $23(15.6 \%)$ & $7(4.7 \%)$ & 140 (94.6\%) & $8(5.4 \%)$ & 0 & 0.000 \\
\hline Weight loss & $\begin{array}{l}<5 \% \\
13(8.8 \%)\end{array}$ & $\begin{array}{l}5 \%-10 \% \\
39(26.3 \%)\end{array}$ & $\begin{array}{l}\geq 10 \% \\
96(64.9 \%)\end{array}$ & $\begin{array}{l}<5 \% \\
16(10.8 \%)\end{array}$ & $\begin{array}{l}5 \%-10 \% \\
47(31.7 \%)\end{array}$ & $\begin{array}{l}\geq 10 \% \\
85(57.5 \%)\end{array}$ & 0.423 \\
\hline Late toxicities & Grade 0 & Grade 1 & Grade 2+ & Grade 0 & Grade 1 & Grade 2+ & \\
\hline Subcutaneous fibrosis & $92(62.2 \%)$ & $53(35.8 \%)$ & $3(2.0 \%)$ & 87 (58.8\%) & $51(34.5 \%)$ & $10(6.7 \%)$ & 0.139 \\
\hline Xerostomia & 29 (19.6\%) & $111(75.0 \%)$ & $8(5.4 \%)$ & 19 (12.8\%) & $122(82.4 \%)$ & $7(4.7 \%)$ & 0.263 \\
\hline Otitis media & $116(78.4 \%)$ & $32(21.6 \%)$ & 0 & $116(78.4 \%)$ & $31(20.9 \%)$ & $1(0.7 \%)$ & 0.602 \\
\hline Taste changes & $106(71.6 \%)$ & $41(27.7 \%)$ & $1(0.7 \%)$ & $120(81.1 \%)$ & $25(16.9 \%)$ & $3(2.0 \%)$ & 0.057 \\
\hline Dehisce difficulty & $122(82.4 \%)$ & $25(16.9 \%)$ & $1(0.7 \%)$ & $132(89.2 \%)$ & $16(10.8 \%)$ & $1(0.7 \%)$ & 0.306 \\
\hline Hearing loss & 75 (50.7\%) & $60(40.5 \%)$ & $13(8.8 \%)$ & $80(54.1 \%)$ & 59 (39.9\%) & $9(6.1 \%)$ & 0.639 \\
\hline Tooth and periodontal diseases & $86(58.1 \%)$ & $56(37.8 \%)$ & $6(4.1 \%)$ & $80(54.1 \%)$ & $52(35.1 \%)$ & $16(10.8 \%)$ & 0.086 \\
\hline Hypothyroidism & $143(96.6 \%)$ & $4(2.7 \%)$ & $1(0.7 \%)$ & $138(93.2 \%)$ & $8(5.4 \%)$ & $2(1.4 \%)$ & 0.416 \\
\hline
\end{tabular}

year. Our centre conducted P70 study with the same fractionation mode and achieved a 3-year LRRFS of 92.7\%. Although this result was consistent with many other studies, we tried to optimize the fractionation pattern. In theory, the best radiation therapy plan should be under the premise of tolerance of OARs to achieve maximum destruction of tumour tissue. Because the regeneration of LRTs is slow and generally not affected by the total time of radiation therapy, the biological effects of radiation to early responding tissues (ERTs) are similar to that of tumour tissues, all ways to improve local control is bound to increase ERT damage. During radiation therapy, acute side-effects occur in oral cavity mucosa, pharyngeo-esophageal mucosa and other ERTs often become the main factors affecting the treatment compliance. The incidence of grade 2-4 oral mucositis was $29.4 \%, 36.8 \%$ and $4.4 \%$, respectively in the RTOG 0225 study. However, with dosimetric advantages and image guided radiation therapy (IGRT) realized with

Table 6 Failure analysis in P67.5 and P70 study

\begin{tabular}{|c|c|c|c|c|c|c|c|c|c|c|}
\hline \multirow[t]{2}{*}{ Failure patterns } & \multicolumn{2}{|c|}{$\begin{array}{l}\text { Num of } \\
\text { patients }\end{array}$} & \multicolumn{2}{|c|}{$\begin{array}{l}\text { Median failure time month } \\
\text { (range) }\end{array}$} & \multicolumn{2}{|c|}{$\begin{array}{l}\text { Num of salvage } \\
\text { treatment }(\%)\end{array}$} & \multicolumn{2}{|c|}{ Num of death (\%) } & \multicolumn{2}{|c|}{$\begin{array}{l}\text { Median time from failure to death } \\
\text { month (range) }\end{array}$} \\
\hline & P67.5 & P70 & P67.5 & P70 & P67.5 & P70 & P67.5 & P70 & P67.5 & P70 \\
\hline Local recurrence & 5 & 6 & $22.0(15-29)$ & $12.8(5-34)$ & $3(60 \%)$ & $2(33 \%)$ & $3(60 \%)$ & $4(67 \%)$ & $10.3(3-18)$ & $4.0(1-7)$ \\
\hline In-field & 3 & 4 & $24.3(21-29)$ & $15.0(6-34)$ & $2(66 \%)$ & $1(25 \%)$ & $2(66 \%)$ & $3(75 \%)$ & $6.5(3-10)$ & $3.6(1-7)$ \\
\hline Marginal & 2 & 2 & $18.5(15-22)$ & $8.5(5-12)$ & $1(50 \%)$ & $1(50 \%)$ & $1(50 \%)$ & $1(50 \%)$ & 18 & 5 \\
\hline Reginal recurrence & 3 & 3 & $25.6(23-30)$ & $16.7(10-24)$ & $3(100 \%)$ & $3(100 \%)$ & 0 & $2(67 \%)$ & - & $12.5(12-13)$ \\
\hline Distant metastasis & 10 & 11 & $10.9(4-26)$ & $19.4(3-38)$ & $5(50 \%)$ & $5(45 \%)$ & $9(90 \%)$ & $9(82 \%)$ & $8.3(3-19)$ & $8.5(0-35)$ \\
\hline Liver & 5 & 3 & $14.8(12-16)$ & $20.3(3-29)$ & $2(40 \%)$ & 0 & $4(80 \%)$ & $3(100 \%)$ & $7.5(3-19)$ & $3.7(1-5)$ \\
\hline Bone & 3 & 4 & $6.3(4-9)$ & $17.5(3-38)$ & $2(67 \%)$ & $2(50 \%)$ & $3(100 \%)$ & $3(75 \%)$ & $9.0(3-13)$ & $13.7(0-35)$ \\
\hline Lung & 1 & 0 & 8 & - & $1(100 \%)$ & - & $1(100 \%)$ & - & 13 & - \\
\hline Multiple or others & 1 & 4 & 8 & $20.5(10-30)$ & 0 & $3(75 \%)$ & $1(100 \%)$ & $3(75 \%)$ & 5 & $8.3(1-17)$ \\
\hline Hemorrhage & 1 & 5 & 10 & $9.2(6-12)$ & 0 & 0 & $1(100 \%)$ & $5(100 \%)$ & 0 & 0 \\
\hline Other patterns & 0 & 1 & - & 12 & - & 0 & - & $1(100 \%)$ & - & 0 \\
\hline
\end{tabular}



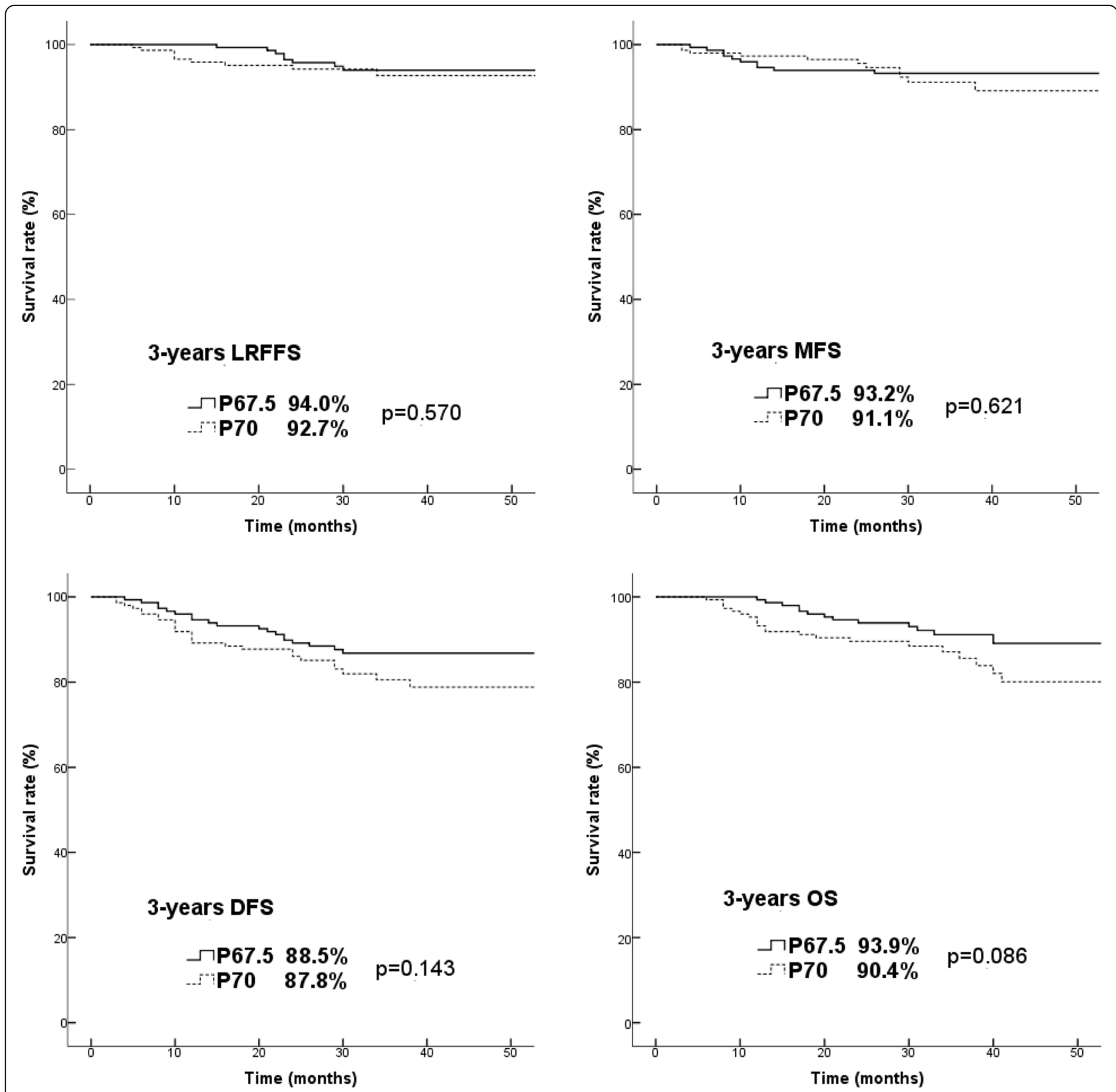

Fig. 1 Kaplan-Meier survival analysis in the propensity-matched cohort of 296 patients. $P$-values were calculated using the log-rank test

megavoltage computed tomography (MVCT) equipped on the gantry, radiation-induced acute injuries in ERT is decreased with HT technique. The incidence of grade 2-3 mucositis and esophageo-tracheitis in P70 group was only $56.8 \%, 3.2 \%$ and $52.1 \%, 0.5 \%$, respectively, without grade 4 side-effects. If the BED remains the same, increased fractional dose and shortened OTT end to a decreased prescription dose, which would result in the following advantages: 1) Improve LCR; Many studies have shown tumour cells appeared accelerated repopulation during the late period of radiation therapy and the total dose should compensate $0.6 \mathrm{~Gy}$ for every extra day of the OTT (equal to $\gamma / \alpha$ value) [12-14], so appropriate shorten the OTT could improve LCR. 2) Reduce dose to OARs; In P67.5 group, maximum doses of brainstem, spinal cord, eyeball, lens, optic nerve and the mean dose of temporomandibular joint, oral cavity, pharyngeo-esophageo-trachea were significantly lower than in P70 group. 3) Reduce costs; The treatment cost reduced by about $3.9 \%$, and the costs of accommodation, food and transportation were correspondingly reduced too. 4) Improve equipment utilization; Physical depreciation of machinery reduces about $9.1 \%$ and the saved medical resources can be used to treat additional 8 patients a year. In P67.5 group, the incidence of acute toxicities such as oral 
Table 7 Univariate analysis with Log-rank test

\begin{tabular}{|c|c|c|c|c|c|c|c|c|c|c|c|c|c|}
\hline \multirow[t]{2}{*}{ Factor } & \multirow[t]{2}{*}{$n$} & \multicolumn{3}{|l|}{ 3-y LRRFS } & \multicolumn{3}{|l|}{ 3-y DMFS } & \multicolumn{3}{|l|}{ 3-y DFS } & \multicolumn{3}{|l|}{$3-y$ OS } \\
\hline & & Events(n) & Survival & $P^{*}$ & Events(n) & Survival & $P^{*}$ & Events(n) & Survival & $P^{*}$ & Events(n) & Survival & $P^{*}$ \\
\hline \multicolumn{14}{|l|}{ Age } \\
\hline$<50$ & 187 & 8 & $96.0 \%$ & 0.127 & 9 & $94.9 \%$ & 0.049 & 19 & $89.2 \%$ & 0.002 & 14 & $91.6 \%$ & 0.008 \\
\hline$\geq 50$ & 109 & 9 & $92.8 \%$ & & 12 & $90.9 \%$ & & 26 & $79.9 \%$ & & 20 & $82.8 \%$ & \\
\hline \multicolumn{14}{|l|}{ Gender } \\
\hline Male & 215 & 10 & $97.1 \%$ & 0.207 & 19 & $93.9 \%$ & 0.057 & 35 & $88.3 \%$ & 0.397 & 27 & $88.0 \%$ & 0.347 \\
\hline Female & 81 & 7 & $91.5 \%$ & & 2 & $97.0 \%$ & & 10 & $87.6 \%$ & & 7 & $89.2 \%$ & \\
\hline \multicolumn{14}{|l|}{ T Stage } \\
\hline $\mathrm{T} 1$ & 88 & 2 & $98.9 \%$ & 0.034 & 6 & $93.1 \%$ & 0.855 & 9 & $88.6 \%$ & 0.045 & 7 & $92.0 \%$ & 0.321 \\
\hline $\mathrm{T} 2$ & 89 & 3 & $96.4 \%$ & & 6 & $94.4 \%$ & & 10 & $89.8 \%$ & & 9 & $93.2 \%$ & \\
\hline T3 & 68 & 8 & $86.2 \%$ & & 4 & $91.9 \%$ & & 13 & $79.8 \%$ & & 10 & $81.3 \%$ & \\
\hline T4 & 51 & 4 & $90.3 \%$ & & 5 & $87.5 \%$ & & 13 & $71.6 \%$ & & 8 & $79.9 \%$ & \\
\hline \multicolumn{14}{|c|}{ Node category } \\
\hline N- & 43 & 2 & $95.0 \%$ & 0.762 & 2 & $94.9 \%$ & 0.537 & 4 & $90.1 \%$ & 0.287 & 4 & $90.5 \%$ & 0.664 \\
\hline $\mathrm{N}+$ & 253 & 15 & $97.2 \%$ & & 19 & $94.8 \%$ & & 41 & $82.9 \%$ & & 30 & $88.0 \%$ & \\
\hline \multicolumn{14}{|l|}{ N Stage } \\
\hline No & 43 & 2 & $95.0 \%$ & 0.625 & 2 & $94.9 \%$ & 0.451 & 4 & $90.1 \%$ & 0.401 & 4 & $90.5 \%$ & 0.876 \\
\hline N1 & 102 & 4 & $95.7 \%$ & & 7 & $92.1 \%$ & & 14 & $85.2 \%$ & & 11 & $90.0 \%$ & \\
\hline N2 & 132 & 9 & $95.1 \%$ & & 12 & $91.1 \%$ & & 25 & $84.6 \%$ & & 17 & $87.1 \%$ & \\
\hline N3 & 19 & 2 & $88.8 \%$ & & 0 & $100.0 \%$ & & 2 & $88.8 \%$ & & 2 & $86.1 \%$ & \\
\hline \multicolumn{14}{|l|}{ UICC Stage } \\
\hline 1 & 15 & 0 & $100.0 \%$ & 0.125 & 0 & $100.0 \%$ & 0.273 & 0 & $100.0 \%$ & 0.019 & 0 & $100.0 \%$ & 0.121 \\
\hline$\|$ & 76 & 1 & $98.6 \%$ & & 3 & $96.0 \%$ & & 5 & $93.3 \%$ & & 5 & $96.0 \%$ & \\
\hline III & 134 & 11 & $92.6 \%$ & & 13 & $90.3 \%$ & & 26 & $83.9 \%$ & & 20 & $84.4 \%$ & \\
\hline IV & 71 & 5 & $91.8 \%$ & & 5 & $90.9 \%$ & & 14 & $78.0 \%$ & & 9 & $84.4 \%$ & \\
\hline \multicolumn{14}{|c|}{ Induction chemotherapy was performed or not in stage III-IVpatients } \\
\hline No & 97 & 8 & $89.8 \%$ & 0.608 & 9 & $88.8 \%$ & 0.650 & 22 & $74.8 \%$ & 0.172 & 18 & $79.9 \%$ & 0.058 \\
\hline Yes & 108 & 8 & $91.5 \%$ & & 9 & $91.5 \%$ & & 18 & $82.6 \%$ & & 11 & $88.3 \%$ & \\
\hline
\end{tabular}

Abbreviation: 3-y LRRFS 3-year local-regional relapse free survival; 3-y DMFS 3-year distant metastasis free survival; 3-y DFS 3-year disease free survival; 3-y OS 3-year overall survival

*P-values were calculated using the unadjusted log-rank test

Table 8 Multivariate analysis with Cox proportional hazard model

\begin{tabular}{|c|c|c|c|c|c|c|c|c|}
\hline \multirow[t]{2}{*}{ Factor } & \multicolumn{2}{|l|}{ 3-year LRRFS } & \multicolumn{2}{|l|}{ 3-year DMFS } & \multicolumn{2}{|l|}{ 3-year DFS } & \multicolumn{2}{|l|}{ 3-year OS } \\
\hline & $\mathrm{HR}(95 \% \mathrm{Cl})$ & $P^{*}$ & HR $(95 \%$ Cl) & $P^{*}$ & $\mathrm{HR}(95 \% \mathrm{Cl})$ & $P^{*}$ & HR $(95 \% \mathrm{Cl})$ & $P^{*}$ \\
\hline Treatment pattern (P67.5 vs.P70) & $0.653(0.249-1.714)$ & 0.387 & $0.682(0.286-1.623)$ & 0.387 & $0.564(0.310-1.024)$ & 0.060 & $0.476(0.236-0.957)$ & 0.037 \\
\hline Gender (female vs. male) & $2.481(0.927-6.644)$ & 0.071 & $0.279(0.065-1.209)$ & 0.088 & $0.878(0.431-1.791)$ & 0.721 & $0.765(0.328-2.411)$ & 0.535 \\
\hline Age ( $\geq 50$ vs. $<50$ years) & $2.672(0.990-7.216)$ & 0.052 & $2.617(1.076-6.364)$ & 0.034 & $3.058(1.659-5.635)$ & 0.000 & $2.914(1.434-5.921)$ & 0.003 \\
\hline T Stage (3-4 vs.1-2) & $2.715(0.784-9.404)$ & 0.115 & $0.391(0.105-1.453)$ & 0.161 & $1.196(0.558-2.562)$ & 0.646 & $0.960(0.382-2.411)$ & 0.931 \\
\hline Node category (N+ vs. N-) & $0.957(0.172-5.328)$ & 0.960 & $1.891(0.389-9.196)$ & 0.430 & $1.856(0.607-5.681)$ & 0.278 & $1.542(0.483-4.925)$ & 0.465 \\
\hline N Stage (2-3 vs. $0-1)$ & $1.423(0.383-5.291)$ & 0.598 & $0.359(0.085-1.515)$ & 0.163 & $0.801(0.351-1.824)$ & 0.597 & $0.691(0.255-1.872)$ & 0.467 \\
\hline UICC Stage (III-IV vs. I-II) & 4.031 (0.338-48.101) & 0.270 & 9.786 (1.448-66.128) & 0.019 & $4.487(1.245-16.166)$ & 0.022 & $4.208(1.026-17.263)$ & 0.046 \\
\hline
\end{tabular}


mucositis and esophageo-tracheitis was $8.8 \%$ and $2.7 \%$, respectively, without significant difference compared to that in P70 study, even with more patients receiving CCRT. All of the above results confirm that the fractionation pattern of $67.5 \mathrm{~Gy} / 30$ was safe and feasible.

Improving the survival rate was still one of the intentions of the P67.5 study. Compared with P70 study, the absolute value of the 3-year LRRFS, DMFS, DFS and OS in P67.5 study was improved by $1.3 \%, 2.1 \%, 0.7 \%$ and $3.5 \%$, respectively. Although statistical significance was not achieved, we observed a trend of improvement in the 3-year OS, which was confirmed by multivariate analysis. Univariate analysis of all cases showed that $\mathrm{T}$ stage was the only factor affecting the LRRFS and increasing the fractional dose did not improve the LCR, but it was known that the good overall outcome of NPC and the use of SMART technology could both result in a good LCR [11, 15-18], so a 3-year LRRFS of $94 \%$ in P67.5 study was acceptable. T stage not only affected LRRFS, but together with UICC stage also affected DFS, which showed that the progression of the disease was closely related to the severity of the primary tumour and the clinical stage.

Despite the LCR has been guaranteed by the wide application of IMRT in NPC, distant metastasis was still the first reason of treatment failure. In recent years, a large number of clinical evidence suggested that CCRT could improve the survival rate of patients with locally advanced NPC and the 5-year DMFS attained up to $74.7 \%-85.8 \%$ [19-21], at the same time anti-EGFR Mab treatment also made clinical benefit in NPC patients $[7,22]$. In both groups, CCRT was the standard treatment for locally advanced NPC patients, while anti-EGFR Mab treatment was also performed and the 3-years DMFS was $92.5 \%$, the same with the literatures, but 21 cases developed distant metastases, almost double number of the cases with locoregional failure. Whether ICT could improve the survival of patients with locally advanced NPC was still controversial, some studies have shown its benefits. The phase II study conducted by Ferrari et al. [23] confirmed that patients with locally advanced NPC received induction regimen of cisplatin and fluorouracil (PF) followed by cisplatin-based CCRT, had improved LCR and OS. Hui et al. [24] added ICT with DP regimen (docetaxel $75 \mathrm{mg} /$ $\mathrm{m}^{2}+$ cisplatin $75 \mathrm{mg} / \mathrm{m}^{2}$ ) and showed a significant improvement of 3-year OS and a trend of improvement of 3year PFS and DMFS compared with CCRT alone regimen (cisplatin $40 \mathrm{mg} / \mathrm{m} 2$ per week). The phase III study conducted by Sun et al. [25] conformed that addition of TPF induction chemotherapy (docetaxel $60 \mathrm{mg} / \mathrm{m}^{2}$, cisplatin $60 \mathrm{mg} / \mathrm{m}^{2}$ intravenously every 3 weeks and fluorouracil $600 \mathrm{mg} / \mathrm{m}^{2}$ per day as a continuous $120 \mathrm{~h}$ infusion) to CCRT significantly improved the 3-year failure-free survival compared with CCRT alone ( $80 \%$ vs. $72 \%, p=0.034$ ) in locoregionally advanced nasopharyngeal carcinoma with acceptable toxicities. Based on the results of the above studies, we were more inclined to use ICT + CCRT regimen hoping to improve the survival and the use rate of ICT + CCRT regimen in P67.5 group was as high as $90.5 \%$, while that in P70 group was only $13.0 \%$. However, there was no statistical significance in 3-year LRRFS, DMFS, DFS and OS between patients with ICT + CCRT regimen and CCRT alone, and the same result was obtained by other recent prospective randomized studies [26-28]. In Xu's study [29], it was found that ICT only improved the DMFS and OS in patients with N3 disease, so what kind of patients with local advanced NPC could benefit from ICT might need more studies. In addition, in our study, age was another factor affecting survival rate and the 3-year DMFS, DFS and OS in patients aged $\geq 50$ years were significantly lower than that in patients aged $<50$ years, which was also shown in Qiu's study [30].

In failure patients of NPC, active salvage therapy might achieve prolonged survival, or even radical cure. Zhou et al. [31] reirradiated 53 locally recurrent patients with IMRT (67.9Gy) combined with cisplatin-based chemotherapy and the 2-year OS and progression-free survival (PFS) were $58.7 \%$ and $52.3 \%$, respectively. Goto et al. [32] reirradiated 50 locally relapsed patients using HT plus concurrent chemotherapy and got similar results. It has been recognized that platinum-based chemotherapy as the first-line treatment achieved an objective response (OR) up to $50-90 \%$ in metastatic NPC [33], and could obtain an OR of $22-75 \%$ even as a second-line treatment [34]. Zheng et al. [35] retrospectively analyzed three kinds of treatment in patients with metastatic NPC and found that salvage chemotherapy plus palliative radiation therapy or other localized treatment resulted in better survival than chemotherapy alone or supportive treatment, and the 2-year DMFS reached to $57.7 \%$, while that in the other two groups was only $32.7 \%$ and $1.6 \%$, respectively. Currently there was no standard treatment for relapsed NPC. Zheng et al. [35] suggested that active salvage therapy should be necessary, and systemic treatment should be combined with local treatment, and local treatment should not be limited to the nasopharynx but extended to the appropriate metastatic lesions. In this study, six regional relapse patients, all received salvage therapy, had the best prognosis with a survival rate as high as $67 \%$. The prognosis of local recurrence was worse, 5 (45\%) of 11 these patients received salvage therapy and 7 cases $(64 \%)$ died. The worst prognosis was happened in distant metastatic patients, 11 cases (48\%) receiving salvage therapy, 18 (86\%) died. The incidence and mortality of the above three failure patterns were comparable in both groups. It was noted that there were 5 patients without loco-regional recurrence or distant metastasis died of hemorrhage in this study, which was rarely reported in the literatures. In the study of Lin et al. [36], among the 370 patients of NPC, only one died 
of local hemorrhage. Nasopharyngeal hemorrhage is one of the common complications after radiation therapy, which is relatively easy to control, and uncontrollable hemorrhage is often associated with local recurrence. At the beginning of the P67.5 study, we realized the importance of nasal care and regular review after radiation therapy and only one patient died of hemorrhagic till now. The difference in this failure pattern between the two groups, led to a significant difference $(p=0.037)$ in the 3year OS analyzed in multivariate analysis.

\section{Conclusions}

Through increasing the fractional dose and shorten the treatment time, the P67.5 study achieved excellent short-term outcomes and potential clinical benefits, with acceptable acute and late toxicities. The long-term outcomes are under investigation.

\begin{abstract}
Abbreviations
ACT: Adjuvant chemotherapy; BED: Biological effective dose; CCRT: concurrent chemoradiotherapy; CR: Complete remission; CTCAE: Common Terminology Criteria for Adverse Events; CTV: Clinical target volume; DFS: Disease free survival; DMFS: Distant metastasis free survival; ERTs: Early responding tissues; GTV: Gross tumor volume; HR: Hazard ratios; ICT: Induction chemotherapy; IGRT: Image guided radiation therapy; IMRT: Intensity modulated radiation therapy; KPS: Karnofsky performance status; LCR: Local control rate; LRRFS: Local-regional relapse free survival; LRTs: Late reaction tissues; MRI: Magnetic resonance imaging; MVCT: Megavoltage computed tomography; NPC: Nasopharyngeal carcinomas; OARs: Organs at risk; OR: Objective response; OS: Overall survival; OTT: Overall treatment time; PET: Positron emission tomography; PFS: Progression-free survival; PR: Partial remission; PSM: Propensity score matching method; PTV: Planning target volume; RTOG/EORTC: Radiation Therapy Oncology Group and the European Organization for Research and Treatment of Cancer; SD: Stable disease; SMART: Simultaneous modulated accelerated radiation therapy; UICC: Union Internationale Contre le Cancer; WHO: World Health Organization
\end{abstract}

\section{Acknowledgements}

Not applicable.

\section{Funding}

The study was supported by Medical \& Health Research Key Projects of Hainan Province, China (No. 2013Key-11), Science \& Technology Innovation Project of Sanya City (No. 2016YW12), and Nursery Foundation of Chinese PLA General Hospital (No. 14KMM34). All funding bodies have no roles in study design, data collection and analysis, and manuscript preparation.

\section{Availability of data and materials}

The datasets used and analysed during the current study are available from the corresponding author on reasonable request.

\section{Authors' contributions}

Conception and design of the study: LD, LCF and LM. Data collection and editing and revision of the manuscript: LD, XXZ, LCF, BLQ, JC, JY, HXL and LM. Analysis and interpretation of the data: LD, SPX and CBX. Writing and revision of the manuscript: $L D$ and $L M$. $L D$ and $X X Z$ contributed equally to this article. All authors read and approved the final manuscript.

\section{Ethics approval and consent to participate}

The trial was approved and consented by the research ethics committee of the Chinese PLA General Hospital (S2014-048-01), and written informed consent was obtained for all patients.

\section{Consent for publication}

The manuscript does not contain data from any individual person and it is not applicable in this section.

\section{Competing interests}

The authors declare that they have no competing interests.

\section{Publisher's Note}

Springer Nature remains neutral with regard to jurisdictional claims in published maps and institutional affiliations.

\section{Author details}

'Department of Radiation Oncology, Chinese PLA General Hospital, 28 Fuxing Road, Beijing 100853, China. ${ }^{2}$ Department of Radiation Oncology, Hainan Branch of Chinese PLA General Hospital, Haitang Bay, Sanya 572000 China. ${ }^{3}$ Department of Otorhinolaryngology, Chinese PLA General Hospital, 28 Fuxing Road, Beijing 100853, China. ${ }^{4}$ Department of Oncology, The first Affiliated Hospital of Xinxiang Medical University, Jiankang Road, Xinxiang 453100, China. ${ }^{5}$ Department of Radiation Oncology, Beijing Xuanwu Hospital affiliated to Capital Medical University, 45 Changchun Street, Beijing 100053, China.

Received: 28 March 2017 Accepted: 22 August 2017

Published online: 29 August 2017

\section{References}

1. Lee N, Xia P, Quivey JM, Sultanem K, Poon I, Akazawa C, et al. Intensitymodulated radiotherapy in the treatment of nasopharyngeal carcinoma: an updated of the UCSF experience. Int J Radiat Oncol Biol Phys. 2002;53(1):12-22.

2. Fiorino C, Dell'Oca I, Pierelli A, Broggi S, Cattaneo GM, Chiara A, et al. Simultaneous integrated boost (SIB) for nasopharynx cancer with helical tomotherapy. A planning study. Strahlenther Onkal. 2007;183(9):497-505.

3. Leung SW, Lee TF. Treatment of nasopharyngeal carcinoma by tomotherapy: five-year experience. Radiat Oncol. 2013; 10.1186/1748-717X-8-107.

4. Du L, Zhang XX, Feng LC, Chen J, Yang J, Liu HX, et al. Treatment of nasopharyngeal carcinoma using simultaneous modulated accelerated radiation therapy via helical tomotherapy: a phase II study. Radiol Oncol. 2016;50(2):218-25.

5. Austin PC. The relative ability of different propensity score methods to balance measured covariates between treated and untreated subjects in observational studies. Med Decis Mak. 2009;29(6):661-77.

6. Du L, Zhang XX, Ma L, Feng LC, Li F, Zhou GX, et al. Clinical study of nasopharyngeal carcinoma treated by helical tomotherapy in China: 5-year outcomes. Biomed Res Int. 2014; 10.1155/2014/980767.

7. Chan TC, Hsu MM, Goh BC, Hui EP, Liu TW, Millward MJ, et al. Multicenter, phase II study of cetuximab in combination with carboplatin in patients with recurrent or metastatic nasopharyngeal carcinoma. J Clin Oncol. 2005;23(15):3568-76.

8. Huang $X D$, Yi JL, Gao L, Xu GZ, Jin J, Yang WZ, et al. Multi-center phase II clinical trial of humanized anti-epidermal factor receptor monoclonal antibody h-R3 combined with radiotherapy for locoregionally advanced nasopharyngeal carcinoma. Chin J Oncol. 2007;29(3):197-201.

9. Lu TX, Zhao C, Chen CY. An open, multicenter clinicalstudy on cetuximab combined with intensity modulated radiotherapy (IMRT) plus concurrent chemotherapy in nasopharyngeal carcinoma (NPC): preliminary report. J Clin Oncol. 2010;28:7s. (suppl; abstr 5577)

10. Mackie TR, Holmes T, Swerdloff S, Reckwerdt P, Deasy JO, Yang J, et al. Tomotherapy: a new concept for the delivery of dynamic conformal radiotherapy. Med Phys. 1993;20(6):1709-19.

11. Lee N, Harris J, Garden AS, Straube W, Glisson B, Xia P, et al. Intensitymodulated radiation therapy with or without chemotherapy for nasopharyngeal carcinoma: Radiation Therapy Oncology Group phase II trial 0225. J Clin Oncol. 2009;27(22):3684-90.

12. Fowler JF. 21 years of biologically effective dose. Br J Radiol. 2010;83(991):554-68.

13. Withers HR, Tayor JM, Maciejewski B. The hazard of accelerated tumor clonogen repopulation during radiotherapy. Acta Oncol. 1988;27(2):131-46.

14. Withers HR. Biologic basis for altered fractionation schemes. Cancer. 1985;55: 2086-95.

15. Lin SJ, Pan JJ, Han L, Zhang X, Liao X, Lu JJ. Nasopharyngeal carcinoma treated with reduced-volume intensity-modulated radiation therapy: report on the 3-year outcome of a prospective series. Int I Radiat Oncol Biol Phys. 2009;75(4):1071-8.

16. Xiao WW, Huang SM, Han F, Wu SX, Lu LX, Lin CG, et al. Local control, survival, and late toxicities of locally advanced nasopharyngeal carcinoma treated by simultaneous modulated accelerated radiotherapy combined with cisplatin concurrent chemotherapy: long-term results of a phase 2 study. Cancer. 2011;117(9):1874-83. 
17. Bakst RL, Lee N, Pfister DG, Zelefsky MJ, Hunt MA, Kraus DH, et al. Hypofractionated dose-painting intensity modulated radiation therapy with chemotherapy for nasopharygeal carcinoma: a prospective trial. Int J Radiat Oncol Biol Phys. 2011;80(1):148-53.

18. Wang RS, Wu F, Lu HM, Wei B, Feng G, Li G, et al. Definitive intensity-modulated radiation therapy for nasopharyngeal carcinoma: long-term outcome of a multicenter prospective study. J Cancer Res Clin Oncol. 2013;139(1):139-45.

19. Wee J, Tan EH, Tai BC, Wong HB, Leong SS, Tan T, et al. Randomized trial of radiotherapy versus concurrent chemoradiotherapy followed by adjuvant chemotherapy in patients with American Joint Committee on Cancer/ International Union against cancer stage III and IV nasopharyngeal cancer of the endemic variety. J Clin Oncol. 2005;23(27):6730-8.

20. Kwong DL, Sham JT, Au GK. Five-year update on a randomized factorial study on concurrent and adjuvant chemotherapy for advanced nasopharyngeal carcinoma. Int J Radiat Oncol Biol Phys. 2006;66:S15-6.

21. Wu X, Huang PY, Peng PJ, Lu LX, Han F, Wu SX, et al. Long-term follow-up of a phase III study comparing radiotherapy with or without weekly oxaliplatin for locoregionally advanced nasopharyngeal carcinoma. Ann Oncol. 2013;24(8):2131-6.

22. Ramakrishnan MS, Eswaraiah A, Crombet T, Piedra P, Saurez G, lyer H, et al. Nimotuzumab, a promising therapeutic monoclonal for treatment of tumors of epithelial origin. MAbs. 2009;1(1):41-8.

23. Ferrari D, Chiesa F, Codecà C, Calabrese L, Jereczek-Fossa BA, Alterio D, et al. Locoregionally advanced nasopharyngeal carcinoma: induction chemotherapy with cisplatin and 5-fluorouracil followed by radiotherapy and concurrent cisplatin: a phase II study. Oncology. 2008;74(3-4):158-66.

24. Hui EP, Ma BB, Leung SF, King AD, Mo F, Kam MK, et al. Randomized phase II trial of concurrent cisplatin-radiotherapy with or without neoadjuvant docetaxel and cisplatin in advanced nasopharyngeal carcinoma. J Clin Oncol. 2009;27(2):242-9.

25. Sun Y, Li WF, Chen NY, Zhang N, Hu GQ, Xie FY, et al. Induction chemotherapy plus concurrent chemoradiotherapy versus concurrent chemoradiotherapy alone in locoregionally advanced nasopharyngeal carcinoma: a phase 3, multicentre, randomized controlled trial. Lancet Oncol. 2016;17(11):1509-20.

26. Lee AW, Ngan RK, Tung SY, Cheng A, Kwong DL, Lu TX, et al. Preliminary results of trial NPC-0501 evaluating the therapeutic gain by changing from concurrent-adjuvant to induction-concurrent chemoradiotherapy, changing from fluorouracil to capecitabine, and changing from conventional to accelerated radiotherapy fractionation in patients with locoregionally advanced nasopharyngeal carcinoma. Cancer. 2015;121(8):1328-38.

27. Fountzilas G, Ciuleanu E, Bobos M, Kalogera-Fountzila A, Eleftheraki AG, Karayannopoulou G, et al. Induction chemotherapy followed by concomitant radiotherapy and weekly cisplatin versus the same concomitant chemoradiotherapy in patients with nasopharyngeal carcinoma: a randomized phase II study conducted by the Hellenic Cooperative Oncology Group (HeCOG) with biomarker evaluation. Ann Oncol. 2012;23(2):427-35.

28. Tan T, Lim WT, Fong KW, Cheah SL, Soong YL, Ang MK, et al. Concurrent chemo-radiation with or without induction gemcitabine, carboplatin, and paclitaxel: a randomized, phase 2/3 trial in locally advanced nasopharyngeal carcinoma. Int J Radiat Oncol Biol Phys. 2015;91(5):952-60.

29. $\mathrm{Xu} T$ T, Shen $\mathrm{CY}, \mathrm{Ou} X \mathrm{XM}, \mathrm{He} X Y$, Ying HM, Hu CS. The role of adjuvant chemotherapy in nasopharyngeal carcinoma with bulky neck lymph nodes in the era of IMRT. Oncotarget. 2016;7(15):21013-22.

30. Qiu WZ, Huang PY, Shi JL, Xia HQ, Zhao C, Cao KJ. Neoadjuvant chemotherapy plus intensity-modulated radiotherapy versus concurrent chemoradiotherapy plus adjuvant chemotherapy for the treatment of locoregionally advanced nasopharyngeal carcinoma: a retrospective controlled study. Chin J Cancer. 2016; 10.1186/s40880-015-0076-9.

31. Zhou J, Ying $H, H u$ C, Wang XG. Preliminary Results of Re-irradiation for Locally Recurrent Nasopharyngeal Carcinoma with Intensity Modulated Radiotherapy. Int J Radiat Oncol Biol Phys. 2010;48(3):S468.

32. Goto Y, Ito J, Tomita N, Kodaira T. Re-irradiation Combined with Concurrent Chemotherapy for Patients with Locally Recurrent Nasopharyngeal Carcinoma: Clinical Advantage of Intensity Modulated Radiotherapy using Helical Tomotherapy. Int J Radiat Oncol Biol Phys. 2010;48(3):S460.

33. Chan AT, Grégoire V, Lefebvre JL, Licitra L, Hui EP, Leung SF, et al. Nasopharyngeal cancer: EHNS-ESMO-ESTRO Clinical Practice Guidelines for diagnosis, treatment and follow-up. Ann Oncol. 2012;23 Suppl 7: vii83-5.
34. Bensouda Y, Kaikani W, Ahbeddou N, Rahhali R, Jabri M, Mrabti H, et al. Treatment for metastatic nasopharyngeal carcinoma. Eur Ann Otorhinolaryngol Head Neck Dis. 2011;128(2):79-85.

35. Zheng W, Zong J, Huang C, Chen J, Wu J, Chen C, et al. Multimodality Treatment May Improve the Survival Rate of Patients with Metastatic Nasopharyngeal Carcinoma with Good Performance Status. PLoS One. 2016; 10.1371/journal.pone.0146771.

36. Lin SJ, Lu JJ, Han L, Chen Q, Pan J. Sequential chemotherapy and intensitymodulated radiation therapy in the management of locoregionally advanced nasopharyngeal carcinoma: Experience of 370 consecutive cases. BMC Cancer. 2010; 10.1186/1471-2407-10-39.

\section{Submit your next manuscript to BioMed Central and we will help you at every step:}

- We accept pre-submission inquiries

- Our selector tool helps you to find the most relevant journal

- We provide round the clock customer support

- Convenient online submission

- Thorough peer review

- Inclusion in PubMed and all major indexing services

- Maximum visibility for your research

Submit your manuscript at www.biomedcentral.com/submit
C Biomed Central 